\title{
Profile of non-compliance in lymphoblastic leukaemia
}

\author{
D Lancaster, L Lennard, J S Lilleyman
}

\begin{abstract}
A nationwide study of intracellular drug metabolite concentrations in children prescribed 6-mercaptopurine for the treatment of lymphoblastic leukaemia was carried out to assess interpatient variability at a standardised dose. Nine children ( $2 \%$ of the total) had completely undetectable metabolites, indicative of noncompliance. Five were adolescents, but otherwise they had no obvious distinguishing characteristics. Not taking any 6-mercaptopurine at all is uncommon, but the problem cannot be predicted. The total number of children who do not comply cannot be determined from this study, but the nine children described represent only a fraction of these.

(Arch Dis Child 1997;76:365-366)
\end{abstract}

Keywords: lymphoblastic leukaemia; compliance; 6-mercaptopurine

An important part of treatment for common childhood acute lymphoblastic leukaemia (ALL) is an extended period, usually two years, of remission 'maintenance' treatment based on antimetabolites taken by mouth. The main drug, 6-mercaptopurine, is taken daily and the dose is titrated to tolerance against toxicity manifest as falling neutrophil and platelet counts, or both. Drug derived metabolites accumulate in cells slowly over several days and disappear on a similar timescale. They can be conveniently assayed in red cells. ${ }^{1}$ The concentration of such metabolites varies from time to time and between patients for several reasons, but a total absence while supposedly ingesting a substantial daily dose of 6-mercaptopurine is hard to explain on any other grounds than not taking the drug. We have encountered a small number of such children from all over the UK and regard them as the tip of the compliance iceberg. We summarise their characteristics and clinical circumstances in this short report.

\section{Patients and methods}

Blood samples from children with ALL receiving 'maintenance' treatment were sent from all over the UK for assays of 6-mercaptopurine metabolites in red blood cells. They were taken at any time when 6-mercaptopurine had been prescribed at full protocol dose (or more) for at least seven days, by which time the intracellular metabolites should have been easily detectable and at or approaching steady state levels. In the small number of children where the assay values were zero, clinical information, includ- ing demographic and social factors, and an assessment of compliance were sought.

\section{Results}

A total of 496 children was studied. The range of metabolite concentrations seen (6thioguanine nucleotides) ranged from 0 to $1410 \mathrm{pmol} / 10^{8}$ red blood cells, with a median value of 301 . Nine children (2\%) were found to have completely undetectable metabolites on one or more occasions. A further four children had less than $50 \mathrm{pmol} / 10^{8}$ (and were thus at the limit of the assay sensitivity), 10 had values between 50 and $100 \mathrm{pmol} / 10^{8}$, and 21 between 100 and $150 \mathrm{pmol} / 10^{8}$. All patients within this range would give cause for concern about the adequacy of treatment.

Of the nine children with a zero concentration of metabolites, six were boys and three girls. They were aged from 5 to 18 years (median 11 years). All but one were white Europeans. Four were from social classes 4 and 5 and two from classes 1 and 2. Of the fathers whose occupations were known, four were drivers (lorry, van, or taxi), and the others included work in business, environmental health, and psychology. Four of the mothers worked outside the home in business, psychology, journalism, and cleaning. Five of the nine children had older siblings, six had younger siblings, and three had both. Five (55\%) were adolescents compared with $83(17 \%)$ of the remaining children $\left(\chi^{2}=6.52, \mathrm{p}<0.02\right)$.

At the time the samples were taken the children had supposedly been taking from 67 to $188 \mathrm{mg} / \mathrm{m}^{2}$ of daily 6 -mercaptopurine without reduction for four to 52 weeks. All but one had completed more than one year of treatment. Their ages and the doctor's assessment of the likelihood and possible reason for noncompliance are shown in table 1 . There was no apparent reason to suspect non-compliance in only one patient. Of the nine children who did not comply with treatment, seven continue in their first remission and two of those are still receiving treatment. The remaining children have been followed for two to four years after the completion of treatment.

\section{Discussion}

An assay of 6-mercaptopurine metabolites in red blood cells has provided a convenient way of monitoring the adequacy of 'maintenance' treatment given by mouth in childhood ALL; those children with lower concentrations are at a higher risk of relapse. ${ }^{12}$ One of many reasons for low concentrations is poor compliance and we have a growing suspicion that this may be a 
Table 1 Patients with undetectable metabolites: doctor's assessment of possible reasons for non-compliance

\begin{tabular}{|c|c|c|c|}
\hline Patient No & Age (years) & $\begin{array}{l}\text { Likely } \\
\text { non-compliant? }\end{array}$ & Possible reason for non-compliance \\
\hline 1 & 12 & Yes & Tablets made her feel ill and were a daily reminder that she had cancer \\
\hline 2 & 5 & Yes & Parents had idiosyncratic views about treatment \\
\hline 3 & 15 & Yes & Lack of motivation/supervision \\
\hline 4 & 7 & Possible & Lack of supervision/chaotic family \\
\hline 5 & 5 & Yes & Difficult family circumstances with frequent social work input \\
\hline 6 & 11 & No & No obvious problem \\
\hline 7 & 14 & Yes & School and behavioural problems. Admitted to not taking tablets \\
\hline 8 & 18 & Yes & $\begin{array}{l}\text { Fed up with treatment, wanted to be 'normal'. Refused bone marrow } \\
\text { transplantation. Was on prolonged maintenance treatment }\end{array}$ \\
\hline 9 & 6 & Yes & $\begin{array}{l}\text { Complex social situation. Family accepted that treatment not given. Maternal } \\
\text { grandmother blamed }\end{array}$ \\
\hline
\end{tabular}

more important problem than is generally appreciated. $^{3}$ More children than the nine described here in detail produced inappropriately low or very low assay values that may well have been the result of intermittent or partial compliance, but alternative explanations cloud the issue. The total absence of metabolites is hard to explain on any other basis, however, so we chose to concentrate on those nine children, imagining them to be representative of the greater population of delinquent compliers.

We wanted to see if they formed any socially or clinically identifiable group, but, apart from there being more adolescents than would be expected by chance, and also that most had been receiving treatment for over a year (both previously suggested risk factors for non-compliance ${ }^{4}$ ), they were hard to categorise. In a previous regional study we noted an excess of children with Asian family names in a group of 32 children with inappropriately low metabolite levels, ${ }^{5}$ but only one of the nine children in this study was Asian. As for outcome, only two of the nine children had relapsed at the time of the study, though the numbers are too small and follow up too short for any conclusions to be drawn from this, except that they must have taken some of their drug some of the time. It may be the duration rather than the degree of non-compliance that is important, with persistent partial defaulters being more at risk than the occasional total abstainer.

We do not know whether the children described here are truly representative of all those who do not reliably take their chemotherapy drugs. All we can say is that adolescents should be watched carefully, particularly in the second year of treatment. More importantly, we emphasise that no social class or ethnic group appears to be immune from the problem and that patients with cancer appear to be no different from other children in this respect.

We are grateful to the clinicians who sent samples and particularly to the following forproviding information about their
patients: Dr V Broadbent, Professor J M Chessells, Dr C Gutteridge, Dr C Haworth, Dr E Hill, Dr J Kernahan, Dr J E Kingston, Dr S Kinsey, Dr D Stevens, and Dr H Wallace.

\footnotetext{
1 Lennard L, Lilleyman JS. Variable mercaptopurine metabolism and treatment outcome in childhood lymphoblastic leukaemia. F Clin Oncol 1989;7:1816-23.

2 Schmiegelow K, Schroder H, Gustaffson G, et al. Risk of relapse in childhood acute lymphoblastic leukemia is related to RBC methotrexate and mercaptopurine metaboites during maintenance chemotherapy. F Clin Oncol 1995; 13:345-51.

3 Lilleyman JS, Lennard L. Non-compliance with oral chemotherapy in childhood leukaemia [editorial]. BMF 1996;313:1219-20.

4 Tebbi CK, Cummings KM, Zevon M, et al. Compliance of pediatric and cancer patients. Cancer 1986;58:1179-84

5 Lennard L, Welch J, Lilleyman JS. Intracellular metabolites of mercaptopurine in children with lymphoblastic leukaemia: a possible indicator of non-compliance? $\mathrm{Br} \mathcal{F}$ Cancer 1995;72:1004-6.
} 